\title{
BOUNDS ON THE HILBERT-KUNZ MULTIPLICITY
}

\author{
OLGUR CELIKBAS, HAILONG DAO, CRAIG HUNEKE, \\ AND YI ZHANG
}

\section{Dedicated to Paul Monsky}

\begin{abstract}
In this paper we give new lower bounds on the Hilbert-Kunz multiplicity of unmixed nonregular local rings, bounding them uniformly away from 1. Our results improve previous work of Aberbach and Enescu.
\end{abstract}

\section{$\S 0$. Introduction}

Let $(R, \mathfrak{m})$ be a $d$-dimensional Noetherian local ring of positive characteristic $p$. For every $m$-primary ideal $I$ in $R$, one can define an asymptotic invariant that measures the singularity of $R$ via the Frobenius powers of $I$. For $q$ a power of $p$, we let $I^{[q]}$ be the ideal generated by the $q$ th powers of all elements of $I$. In 1983, Monsky [M] proved that there is a real number $e_{H K}(I) \geq 1$ such that for $q=p^{e}$,

$$
\ell\left(R / I^{[q]}\right)=e_{H K}(I) q^{d}+O\left(q^{d-1}\right) .
$$

When $I=\mathfrak{m}$, we set $e_{H K}(\mathfrak{m})=e_{H K}(R)$. We use $\ell(\cdot)$ to denote the length of a module, and $\mu(\cdot)$ to denote the minimal number of generators of a module throughout this paper.

Later Huneke, McDermott, and Monsky [HMM] extended this result in the case $R$ is integrally closed and excellent with perfect residue field, to show that there is a constant $\beta$ such that

$$
\ell\left(R / I^{[q]}\right)=e_{H K}(I) q^{d}+\beta q^{d-1}+O\left(q^{d-2}\right) .
$$

See $[\mathrm{HoY}]$ for further refinements.

Received February 16, 2011. Revised April 19, 2011. Accepted August 23, 2011.

2010 Mathematics Subject Classification. Primary 13A35; Secondary 13B22, 13H15, $14 \mathrm{~B} 05$.

Huneke's work partially supported by National Science Foundation grant DMS1063538.

Dao's work partially supported by National Science Foundation grant DMS0834050. 
The number $e_{H K}(R)$ is called the Hilbert-Kunz multiplicity of $R$. If $R$ is regular, it is easy to see that $e_{H K}(R)=1$. The converse is true if $R$ is unmixed, but this is highly nonobvious. In fact, it was not until 2000 that Watanabe and Yoshida [WY1] proved this fact. A somewhat simpler proof can be found in $[\mathrm{HY}]$.

In this paper we are interested in giving lower bounds on the HilbertKunz multiplicity. Let $e$ denote the usual Hilbert-Samuel multiplicity of $R$. It is not hard to prove the following bounds:

$$
\max \left\{\frac{e}{d !}, 1\right\} \leq e_{H K}(R) \leq e .
$$

The last inequality, for example, follows at once from Lech's lemma (see [SH, 11.2.10]).

A natural way to think about the Hilbert-Kunz multiplicity is that as it nears 1, the singularities of the ring should be better. Blickle and Enescu $[\mathrm{BE}]$ were able to prove an explicit version of this principle.

Theorem 0.1 ([BE, Theorems 2.5, 2.7]). Let $(R, \mathfrak{m}, k)$ be an unmixed local ring of prime characteristic $p>0$.

(1) If $e_{H K}(R)<1+1 / d$ !, then $R$ is Cohen-Macaulay and F-rational.

(2) If $e_{H K}(R)<1+1 / p^{d} d$ !, then $R$ is regular.

A Noetherian local ring $R$ of prime characteristic $p$ is said to be F-rational if ideals generated by systems of parameters are tightly closed. For details concerning the theory of tight closure and its relationship to the HilbertKunz multiplicity, we refer to $[\mathrm{HH}]$ and $[\mathrm{H}]$. We will not be directly using tight closure in this paper, except at the very end of the paper.

The second result in Theorem 0.1 is unsatisfactory because of its dependence on $p$. Blickle and Enescu $[\mathrm{BE}]$ raised the question of whether the Hilbert-Kunz multiplicity is uniformly bounded away from 1 for unmixed local rings which are not regular. Specifically, the following was asked.

QUESTION 0.2 (see $[\mathrm{BE}]$ ). Is there a constant $\epsilon(d)$, depending only on the dimension $d$ of $R$, such that if $R$ is an unmixed local ring which is not regular, then $e_{H K}(R)-1 \geq \epsilon(d)$ ?

As a further question, Watanabe and Yoshida asked that if one can determine the infimum of Hilbert-Kunz multiplicities over all unmixed local Noetherian rings of characteristic $p$ and dimension $d$ which are not regular, and if that infimum is attained, how to classify those rings which have the lowest value. 
Aberbach and Enescu [AE] gave a positive answer to Question 0.2. Our main result, Theorem 3.7, improves upon their bound.

One could extend these questions to ask whether the Hilbert-Kunz multiplicities of rings of fixed characteristic and dimension are discrete within any bounded region, or are there limit points?

Question 0.2 was made even more explicit in [WY2], where the following conjecture is posed.

Conjecture 0.3 ([WY2, Conjecture 4.2]). Let $d \geq 1$ be an integer, and let $p>2$ be a prime number. Set $A_{p, d}:=K\left[\left[X_{0}, X_{1}, \ldots, X_{d}\right]\right] /\left(X_{0}^{2}+\cdots+\right.$ $\left.X_{d}^{2}\right)$, where $K$ is the algebraic closure of the field with $p$ elements. Let $(R, \mathfrak{m}, K)$ be a d-dimensional unmixed local ring. Then the following two statements hold. (1) If $R$ is not regular, then $e_{H K}(R) \geq e_{H K}\left(A_{p, d}\right) \geq 1+$ $c_{d} / d$ !. (2) If $e_{H K}(R)=e_{H K}\left(A_{p, d}\right)$, then $\widehat{R} \cong A_{p, d}$.

In this conjecture, $c_{d}$ is defined by the equation

$$
\sec (x)+\tan (x)=\sum_{d=0}^{\infty} \frac{c_{d}}{d !} x^{d}
$$

Gessel and Monsky [GM, Theorem 3.8] proved that $\lim _{p \rightarrow \infty} e_{H K}\left(A_{p, d}\right)=$ $1+c_{d} / d$ !. To get a feeling for what the conjecture says for small dimensions, the coefficients $c_{d} / d$ ! are as follows: $c_{2} / 2 !=1 / 2, c_{3} / 3 !=1 / 3, c_{4} / 4 !=5 / 24$, and $c_{5} / 5 !=2 / 15$. Enescu and Shimomoto $[\mathrm{ES}]$ prove the conjecture for complete intersections.

In [WY2] and [WY3] both problems of lower bounds and of classification are solved in dimensions at most 4. Moreover, they showed that in low dimensions there is a minimal value for the Hilbert-Kunz multiplicity for nonregular rings, and rings which take this value are classified. For example, they proved that if $(R, \mathfrak{m}, k)$ is a two-dimensional Cohen-Macaulay local ring of prime positive characteristic $p$, algebraically closed residue field, and multiplicity $e$, then $e_{H K}(R) \geq(e+1) / 2$ with equality if and only if the associated graded ring of $R$ is isomorphic to the eth Veronese subring of $k[x, y]$. They also proved that if $(R, \mathfrak{m}, k)$ is a three-dimensional unmixed local ring of positive prime characteristic $p$ which is not regular, then $e_{H K}(R) \geq 4 / 3$. Furthermore, if $k$ is algebraically closed of characteristic not 2 , then equality occurs if and only if $R$ is analytically isomorphic with $k[[x, y, z, w]] /\left(x^{2}+y^{2}+z^{2}+w^{2}\right)$. 
Using an intricate and beautiful argument, Aberbach and Enescu solved the basic problem of bounds independent of the characteristic in all dimensions.

Theorem 0.4 ([AE, Theorem 4.12]). Let $(R, \mathfrak{m}, k)$ be an unmixed ring of characteristic $p$ and dimension $d \geq 2$. If $R$ is not regular, then

$$
e_{H K}(R) \geq 1+\frac{1}{d(d !(d-1)+1)^{d}}
$$

Our main results in this paper build from the work of Aberbach and Enescu. ${ }^{*}$ In the next section we give a bound in the Gorenstein case, which is often better than bounds in [AE]. Our improvement comes by introducing the $F$-signature into the proofs and consideration of a certain dual sequence. However, our main theorem, in Section 3, is an improvement of Theorem 0.4, as follows.

THEOREM 0.5. Let $(R, \mathfrak{m}, k)$ be a Noetherian local unmixed ring of prime characteristic $p$ with infinite perfect residue field and dimension $d \geq 2$. Let $\boldsymbol{x}$ be a minimal reduction of $\mathfrak{m}$, and let $\mu$ be the maximal number of minimal generators of any ideal in $R /(\boldsymbol{x})$. Let $t$ be the largest integer such that $\overline{\mathfrak{m}^{t}}$ is not contained in $(\boldsymbol{x})$. If $R$ is not regular, then

$$
e_{H K}(R) \geq 1+\left(\min \left\{\frac{1}{d !},\left(\frac{\mu}{e-\mu}\right) \cdot \frac{1}{\left(\left\lceil\frac{d}{t}\right\rceil\right)^{d}}\right\}\right) .
$$

Here and throughout the paper, by $\bar{J}$ we denote the integral closure of an ideal $J$. Though the bound in Theorem 0.5 is a substantial improvement on Theorem 0.4 , our bound is still probably far from best, as suggested by Conjecture 0.3. However, the method we use has some interest in its own right and may be useful in other contexts. For instance, in Proposition 2.3, we use these ideas to give an affirmative answer to an old conjecture of Watanabe in many cases.

\section{$\S 1$. New estimates}

In this section we give new bounds on the Hilbert-Kunz multiplicity which depend upon the $F$-signature. We first recall that a Noetherian ring $R$ of

\footnotetext{
*As this paper was being completed, Aberbach and Enescu posted on the ArXiv a new paper with better bounds than their previous work. We will compare these bounds to ours in Section 3; our bounds given in Theorem 0.5 are better in general. (See Remark 3.9).
} 
prime characteristic $p$ is said to be $F$-finite if $R$ is a finitely generated $R$ module via the Frobenius.

Definition 1.1. Let $(R, \mathfrak{m}, k)$ be a Noetherian local ring of prime characteristic $p$ and dimension $d$. Assume that $R$ is $F$-finite and reduced. For $q=p^{e}$, write $R^{1 / q} \cong R^{a_{q}} \oplus M$, where $M$ has no free summands. Set $\alpha(R)=$ $\log _{p}\left[k: k^{p}\right]$. Consider the sequence $\left\{a_{q} /\left(q^{d+\alpha(R)}\right)\right\}$. We denote by $s_{-}(R)$ and $s^{+}(R)$ the liminf and limsup, respectively, of the above sequence as $q \rightarrow \infty$. If $s_{-}(R)=s^{+}(R)$, then the limit, denoted by $s(R)$, is called the $F$-signature of $R$.

Throughout the rest of the paper we will write $s$ for $s(R)$ if there is no ambiguity about the ring $R$.

In very recent and striking work, Kevin Tucker $[\mathrm{T}]$ has shown that the F-signature exists in general. In our main technical results the ring will be Gorenstein; in such a case the existence of the $F$-signature is known and easy to prove. For the statement of our first result, Theorem 1.2, it is worth noting that the $F$-signature is always at most 1 and is equal to 1 if and only if the ring is regular (see [HL, Corollary 16]). Thus, the fraction appearing in this theorem is always at least 1 and is only exactly 1 when the ring is regular.

ThEOREM 1.2. Suppose that $(R, \mathfrak{m}, k)$ is a reduced F-finite Gorenstein local ring of prime characteristic $p$ with infinite perfect residue field. Let $e$ denote the multiplicity of $R$, and let $s$ denote the F-signature of $R$. We choose a minimal reduction $\boldsymbol{x}$ of the maximal ideal. For any ideal $I$ of $R$ such that $I \supseteq(\boldsymbol{x})$, let $\mu=\mu(I /(\boldsymbol{x}))$; then

$$
e_{H K}(R) \geq \frac{e-s \mu}{e-\mu}=1+\frac{\mu}{e-\mu}(1-s) .
$$

Proof. We write $e_{H K}$ for $e_{H K}(R)$. Let $F=R^{\mu\left(R^{1 / q}\right)}$. In the proof of this theorem we use the notation $M^{*}=\operatorname{Hom}_{R}(M, R)$. Note that $\left(R^{1 / q}\right)^{*} \cong R^{1 / q}$ since $R$ is Gorenstein: the dual of $R^{1 / q}$ is isomorphic to the canonical module of $R^{1 / q}$, and since $R^{1 / q}$ is also Gorenstein, this canonical module is isomorphic to the ring. Hence, there is a short exact sequence $0 \rightarrow N \rightarrow F \rightarrow$ $\left(R^{1 / q}\right)^{*} \rightarrow 0$. Its dual is exact since $\left(R^{1 / q}\right)^{*}$ is maximal Cohen-Macaulay and $R$ is Gorenstein:

$$
0 \rightarrow R^{1 / q} \rightarrow F^{*} \rightarrow N^{*} \rightarrow 0
$$


(see $\left[\mathrm{BH}\right.$, Theorem 3.3.10(c)]). We now write $R^{1 / q} \cong R^{a_{q}} \oplus M$, where $M$ has no free summand. Note that this means that $a_{q}$ copies of $R$ split from the above short exact sequence, giving rise to an exact sequence

$$
0 \rightarrow M \rightarrow G^{*} \rightarrow N^{*} \rightarrow 0
$$

where $G=R^{\mu\left(R^{1 / q}\right)-a_{q}}$. Observe that the image of $M$ is contained in $\mathfrak{m} G^{*}$ since $M$ has no free summand (if not, then dualizing would give a free summand of $M^{*}$, and hence of $\left.M^{* *} \cong M\right)$.

Since $\mu(F)=\ell\left(R / \mathfrak{m}^{[q]}\right)=e_{H K} q^{d}+O\left(q^{d-1}\right)$, we can write $G^{*}=$ $R^{e_{H K} q^{d}-a_{q}+d_{q}}$, where $d_{q}=O\left(q^{d-1}\right)$. Since $N$ is a maximal Cohen-Macaulay module (MCM), so is $N^{*}$ by $\left[\mathrm{BH}\right.$, Theorem 3.3.10(d)]. Then $\operatorname{Tor}_{1}^{R}\left(N^{*}, R /\right.$ $(\boldsymbol{x}))=0$. Therefore, we have an injection

$$
\frac{M}{\boldsymbol{x} M} \hookrightarrow \frac{G^{*}}{\boldsymbol{x} G^{*}}
$$

Then

$$
\frac{I R^{1 / q}}{\boldsymbol{x} R^{1 / q}}=\frac{I R^{a_{q}}}{\boldsymbol{x} R^{a_{q}}} \oplus \frac{I M}{\boldsymbol{x} M} \hookrightarrow \frac{I R^{a_{q}}}{\boldsymbol{x} R^{a_{q}}} \oplus \frac{(\mathfrak{m} I+\boldsymbol{x}) G}{\boldsymbol{x} G} .
$$

Computing the length, we see that

$$
\begin{aligned}
\ell\left(I R^{1 / q} / \boldsymbol{x} R^{1 / q}\right) & =\ell\left(I^{[q]} R / \boldsymbol{x}^{[q]} R\right) \\
& =\ell\left(R / \boldsymbol{x}^{[q]} R\right)-\ell\left(R / I^{[q]}\right) \\
& \leq a_{q} \ell(I /(\boldsymbol{x}))+\ell((\mathfrak{m} I+(\boldsymbol{x})) /(\boldsymbol{x}))\left(e_{H K} q^{d}-a_{q}+d_{q}\right) .
\end{aligned}
$$

Since $R$ is Cohen-Macaulay, we obtain that

$$
\begin{aligned}
\ell\left(R / \boldsymbol{x}^{[q]} R\right) & =e q^{d} \\
& \leq \ell\left(R / I^{[q]}\right)+a_{q} \ell(I /(\boldsymbol{x}))+\ell((\mathfrak{m} I+(\boldsymbol{x})) /(\boldsymbol{x}))\left(e_{H K} q^{d}-a_{q}+d_{q}\right) .
\end{aligned}
$$

Let $I \subset J_{1} \subset J_{2} \subset \cdots \subset R$ be a cyclic filtration of ideals of $R$ such that $J_{i+1}=J_{i}+\left(s_{i}\right)$ and such that $J_{i+1} / J_{i} \cong k$. Since there are surjections $R / \mathfrak{m}^{[q]} \rightarrow J_{i+1}^{[q]} / J_{i}^{[q]}$, we see that $\ell\left(R / I^{[q]}\right) \leq \ell\left(R / \mathfrak{m}^{[q]}\right) \cdot \ell(R / I)$. Therefore, taking the $q$ th Frobenius of the filtration of $I \subset R$, we get

$$
\begin{aligned}
e q^{d} & \leq \ell\left(R / I^{[q]}\right)+a_{q} \ell(I /(\boldsymbol{x}))+\ell\left(\frac{\mathfrak{m} I+(\boldsymbol{x})}{(\boldsymbol{x})}\right)\left(e_{H K} q^{d}-a_{q}+d_{q}\right) \\
& \leq \ell\left(R / \mathfrak{m}^{[q]}\right) \ell(R / I)+a_{q} \mu+e_{H K} q^{d} \ell\left(\frac{m I+(\boldsymbol{x})}{(\boldsymbol{x})}\right)+d_{q} \ell\left(\frac{m I+(\boldsymbol{x})}{(\boldsymbol{x})}\right) .
\end{aligned}
$$


(Note that $a_{q} \mu=a_{q} \ell(I /(\mathfrak{m} I+(\boldsymbol{x})))$.) Dividing by $q^{d}$ and taking limits as $q \rightarrow \infty$, and observing that $\lim \left(a_{q} / q^{d}\right)=s$ and $\ell(R /(\boldsymbol{x}))=e$, we see that $e \leq e_{H K}(e-\mu)+s \mu$; hence,

$$
\frac{e-s \mu}{e-\mu} \leq e_{H K} .
$$

This result can be used to recover and sometimes improve estimates in [AE]. For example, compare the following to [AE, Corollary 3.7].

Corollary 1.3. Let $(R, \mathfrak{m}, k)$ be a reduced F-finite Gorenstein local ring of prime characteristic $p$ with infinite perfect residue field. Then

$$
e_{H K}(R) \geq \frac{e-s(v-d)}{e-v+d},
$$

where $v$ is the embedding dimension of $R$, that is, the number of minimal generators of $\mathfrak{m}$. If $R$ is not F-regular, then

$$
e_{H K}(R) \geq \frac{e}{e-v+d} .
$$

Proof. The proof of the first statement follows immediately from Theorem 1.2 by applying this theorem to the ideal $I=\mathfrak{m}$. The second statement holds since if $R$ is not $F$-regular, then $s=0$ [HL]. (The converse is also true; see $[\mathrm{AL}]$.)

REMARK 1.4. If $R$ is not $F$-rational (or even not strongly $F$-regular), then $s(R)=0$, and Corollary 1.3, already in [AE] in this case, gives a very good uniform bound away from 1 for the Hilbert-Kunz multiplicity. In fact, when $R$ is Gorenstein, $s(R)>0$ if and only if $R$ is $F$-rational (equivalently in this case, strongly $F$-regular, weakly $F$-regular, or $F$-regular).

In general, even in the $F$-rational case, we would have a very good estimate bounding the Hilbert-Kunz multiplicity away from 1, if we could bound $s$ below 1 uniformly. In fact, the problem of bounding the $F$-signature below 1 and that of bounding the Hilbert-Kunz multiplicity above 1 are in some sense the same problem. In [HL, Proposition 14], the following upper bound is given:

$$
(e-1)(1-s) \geq e_{H K}(R)-1 .
$$

Combining this with Theorem 1.2 and rewriting gives

$$
\frac{\mu}{e-\mu} \leq \frac{e_{H K}(R)-1}{1-s} \leq e-1 .
$$


This means that the ratio in the middle of the two terms we are interested in is trapped between numbers depending on the usual multiplicity of the ring and the number $\mu$. Note that equality holds between the top and bottom terms in the inequality if and only if $\mu=e-1$ if and only if $R$ has minimal multiplicity.

\section{$\S 2$. Chains of integrally closed ideals}

We begin this section with a seemingly unrelated result. The idea behind this is found in the dissertation of Choi [Ch]. Note that we do not assume $R$ to be of prime characteristic.

Proposition 2.1. Let $(R, \mathfrak{m})$ be a local Noetherian domain, and let $I=$ $(J, u)$, where $J$ is an integrally closed $\mathfrak{m}$-primary ideal of $R$ and $u \in J: \mathfrak{m}$. If $M$ is a finitely generated torsion-free $R$-module, then

$$
\ell(I M / J M) \geq \operatorname{rank} M .
$$

Proof. Set $N=(J M: M u)$. Since $M / N \cong((J, u) M) / J M$ and $\mathfrak{m} M \subseteq N$, we can write $M=N+N^{\prime}$ with $\mu\left(N^{\prime}\right)=\ell((I M) /(J M))$. Thus, it suffices to prove that $\mu\left(N^{\prime}\right) \geq \operatorname{rank}(M)$. Since $u\left(M / N^{\prime}\right) \subseteq J\left(M / N^{\prime}\right)$, it follows from the determinantal trick (see [SH, Lemma 2.1.8]) that there is an element $r=u^{n}+j_{1} \cdot u^{n-1}+\cdots+j_{n}$ with $j_{i} \in J^{i}$ for all $i$ such that $r M \subseteq N^{\prime}$. Observe that $r \neq 0$ since $J$ is integrally closed and $u \notin J$. Since $M_{r}=N_{r}^{\prime}$, this implies that $\mu\left(N^{\prime}\right) \geq \operatorname{rank}\left(N^{\prime}\right)=\operatorname{rank}(M)$.

Given two ideals $I$ and $J$ with $J \subseteq I, \bar{\ell}(I / J)$ will denote the length of the longest chain of integrally closed ideals between $J$ and $I$.

Corollary 2.2. Let $(R, \mathfrak{m})$ be a Noetherian local domain. Let $J$ be an integrally closed $\mathfrak{m}$-primary ideal of $R$, and let $I$ be an ideal containing $J$. If $M$ is a finitely generated torsion-free $R$-module, then

$$
\ell(I M / J M) \geq \bar{\ell}(I / J) \cdot \operatorname{rank}(M) .
$$

Proof. Set $n=\bar{\ell}(I / J)$. Then there is a chain of ideals

$$
J=K_{0} \subset K_{1} \subset \cdots \subset K_{n-1} \subset K_{n} \subset I
$$

with $\overline{K_{i}}=K_{i}$ for all $i$. Then

$$
\ell(I M / J M) \geq \sum_{j=0}^{n} \ell\left(K_{j+1} M / K_{j} M\right) \geq \sum_{j=0}^{n} \ell\left(\left(K_{j}, u_{j}\right) M / K_{j} M\right)
$$


for some $u_{j} \in K_{j+1} \cap \operatorname{Soc}\left(K_{j}\right)$. Thus, the result follows from Proposition 2.1.

In [WY1, Conjecture 2.17], the following conjecture was raised.

Let $A$ be a Cohen-Macaulay local ring of characteristic $p>0$. Then for any $\mathfrak{m}$-primary ideal $I$, we have (1) $e_{H K}(I) \geq \ell(A / I)$; (2) if $p d_{A}(A / I)<\infty$, then $e_{H K}(I)=\ell(A / I)$.

Although this conjecture has turned out to not be true (see, e.g., $[\mathrm{Ku}]$ ), our next result shows that (1) is true for many $\mathfrak{m}$-primary ideals.

Proposition 2.3. Assume that $(R, \mathfrak{m})$ is an excellent normal ring of prime characteristic $p$ with an algebraically closed residue field.

(i) If $I$ is an integrally closed $\mathfrak{m}$-primary ideal of $R$, then

$$
e_{H K}(I) \geq \ell(R / I)+e_{H K}(R)-1
$$

(ii) If $I$ is an $\mathfrak{m}$-primary ideal such that there is an integrally closed ideal $K \subset I$ with $\ell(I / K)=1$, then

$$
e_{H K}(I) \geq \ell(R / I)
$$

Proof. (i) From [W, Theorem 2.1], we obtain that there is a composition series $I=I_{0} \subset I_{1} \subset \cdots \subset I_{l-1}=\mathfrak{m} \subset R$ such that $\bar{I}_{i}=I_{i}$ for all $i$. It follows from Corollary 2.2 that

$$
\ell\left(\frac{\mathfrak{m} R^{1 / q}}{I R^{1 / q}}\right) \geq \ell(\mathfrak{m} / I) \cdot \operatorname{rank}\left(R^{1 / q}\right) .
$$

Therefore,

$$
\ell\left(\frac{R^{1 / q}}{I R^{1 / q}}\right) \geq \ell(\mathfrak{m} / I) \cdot \operatorname{rank}\left(R^{1 / q}\right)+\ell\left(\frac{R^{1 / q}}{\mathfrak{m} R^{1 / q}}\right) .
$$

Dividing by $q^{d}$, we get

$$
e_{H K}(I) \geq \ell(R / I)+e_{H K}(R)-1
$$

(ii) Note that $\ell\left(R / K^{[q]}\right)=\ell\left(R / I^{[q]}\right)+\ell\left(I^{[q]} / K^{[q]}\right)$. On the other hand, $\ell\left(I^{[q]} / K^{[q]}\right) \leq \ell\left(R / \mathfrak{m}^{[q]}\right)$. Hence, $\ell\left(R / I^{[q]}\right)+\ell\left(R / \mathfrak{m}^{[q]}\right) \geq \ell\left(R / K^{[q]}\right)$. Dividing by $q^{d}$ and taking limits, we obtain that

$$
e_{H K}(I)+e_{H K}(R) \geq e_{H K}(K) \geq \ell(R / K)+e_{H K}(R)-1
$$

by the first part of the theorem. The result follows. 
Watanabe and Yoshida [WY1, Theorem 2.7] raised the following question: if $(R, \mathfrak{m})$ is an unmixed local ring of prime characteristic $p$, then is it true that for every $\mathfrak{m}$-primary integrally closed ideal $I, e_{H K}(I) \geq \ell(R / I)$ ? Furthermore, if equality holds for some $I$, does it force $R$ to be regular?

Obviously, the first part of this question is answered in the positive by Proposition 2.3 above, provided that $R$ is in addition excellent normal with algebraically closed residue field. We are grateful to the referee for providing the following extension, which also answers positively the second question.

Corollary 2.4. Let $(R, \mathfrak{m})$ be an excellent local analytically irreducible domain of prime characteristic $p$ with an algebraically closed residue field. If $I$ is an integrally closed $\mathfrak{m}$-primary ideal, then $e_{H K}(I) \geq \ell(R / I)$, with equality if and only if $R$ is regular.

Proof. If $R$ is normal, the inequality is given in Proposition 2.3. The normalization $S$ of $R$ is an excellent normal local domain, and it is a finitely generated $R$-module. Let $\mathfrak{n}$ be the maximal ideal of $S$. Then $S / \mathfrak{n}=R / \mathfrak{m}$. In particular, the length of an $S$-module is the same as the length of the same module viewed as an $R$-module, so in computing length we don't need to specify which ring we are working over. Set $L=\overline{I S}$. Then $L$ is an integrally closed ideal of $S$, and $L \cap R=I$. Thus, we have that $e_{H K}(I)=e_{H K}(I S) \geq$ $e_{H K}(L) \geq \ell(S / L)$ by Proposition 2.3 , and $\ell(S / L) \geq \ell(R / I)$, since $R / I \subset$ $S / L$.

For the last claim, suppose first that $R$ is normal but not regular. Then $e_{H K}(I)>\ell(R / I)$ by Proposition 2.3. If $R$ is not normal and we have equality, then equality must occur in all the inequalities in the paragraph above. Then $S$ is regular, and $e_{H K}(I S)=e_{H K}(L)$. By basic facts of tight closure theory (see $[\mathrm{HH}]$ ), it follows that $I S=L$. Moreover, from the same set of inequalities, we must have that $R / I=S / L$. Thus, $S=R+I S$ is an $R$ module, and Nakayama's lemma gives that $R=S$ is regular.

\section{§3. Main result}

Aberbach and Enescu improved the Blickle-Enescu Theorem 0.1(2) by proving the following.

THEOREM 3.1 ([AE]). Let $(R, \mathfrak{m}, k)$ be an unmixed ring of prime characteristic $p$ and dimension $d \geq 2$. If $e_{H K}(R) \leq 1+\max \{1 / d$ !, $1 / e\}$, then $R$ is Gorenstein and F-regular. 
In particular, for the purposes of bounding the Hilbert-Kunz multiplicity away from 1, we may assume that $R$ is Gorenstein and $F$-regular. Recall that $F$-regular means that every ideal in every localization of $R$ is tightly closed. (For the definition of tight closure and basic properties, see $[\mathrm{H}]$. We will use that $F$-regular rings are Cohen-Macaulay and normal.)

We will use the following fact, which appears in [WY1, Theorem 2.7], for example.

THEOREM 3.2. Let $(R, \mathfrak{m}) \rightarrow(S, \mathfrak{n})$ be a module-finite extension of Noetherian local domains. Then for every $\mathfrak{m}$-primary ideal $I$ of $R$,

$$
e_{H K}(I) \cdot[Q(S): Q(R)]=e_{H K}(I S) \cdot[S / \mathfrak{n}: R / \mathfrak{m}],
$$

where $Q(\cdot)$ denotes the field of fractions of a domain.

We also need the following discussion, which is found in [AE, Remark 4.3].

Discussion 3.3. Let $(R, \mathfrak{m})$ be a local domain. Let $z \in \mathfrak{m}$, and let $n$ be a positive integer. Let $y \in R^{+}$be any root of $f(X)=X^{n}-z$. We call $S=R[y]$ a radical extension for the pair $R$ and $z$.

Whenever $S$ is radical for $R$ and $z$, then $b:=[Q(S): Q(R)] \leq n$. Assume also that $R$ is normal and that $z$ is a minimal generator of $\mathfrak{m}$. Then, in fact, $b=n$. Moreover, $S=R[y] \cong R[X] /\left(X^{n}-z\right)$, so that if $R$ is Cohen-Macaulay (resp., Gorenstein), then so is $S$. Also, $S$ is local with maximal ideal $(\mathfrak{m}, y)$, because $\mathfrak{m}$ is certainly in the Jacobson radical of $S$ as $S$ is a finite extension of $R$, and $S / \mathfrak{m} S \cong k[X] /\left(X^{n}\right)$, where $k$ is the residue field of $R$.

Proposition 3.4. Assume that $(R, \mathfrak{m})$ is Cohen-Macaulay and normal, and let $x \in \mathfrak{m}-\mathfrak{m}^{2}$ be part of a minimal reduction of $\mathfrak{m}$. Let $S=R[y]$ with $y^{n}=x$. Then $\mathfrak{m} S+\left(y^{i}\right)$ is integrally closed for any nonnegative integer $i$.

Proof. Assume that the claim is wrong. We may assume that $i \leq n$. Since $S / \mathfrak{m} S \cong k[X] /\left(X^{n}\right)$, the ideals in $S / \mathfrak{m} S$ are linearly ordered, and thus $y^{i-1} \in$ $\overline{\mathfrak{m} S+\left(y^{i}\right)}$. By the valuative criterion (see [SH, Theorem 6.8.3]), $\overline{\mathfrak{m} S+\left(y^{i}\right)}=$ $\bigcap\left(\mathfrak{m} S+\left(y^{i}\right)\right) V \cap R$, where $(V, v)$ runs over all discrete valuation domains of the field of fractions of $R$ centered on the maximal ideal of $R$. Therefore,

$$
v\left(y^{i-1}\right) \geq v\left(\mathfrak{m} S+\left(y^{i}\right)\right) \geq \min \left\{v(\mathfrak{m} S), v\left(y^{i}\right)\right\},
$$

which is equivalent to

$$
(i-1) v(y) \geq \min \{v(\mathfrak{m} S), i v(y)\} .
$$


Hence, $(i-1) v(y) \geq v(\mathfrak{m} S)$; that is, $y^{i-1} \in \overline{\mathfrak{m} S}$. By [SH, Corollary 6.8.12], this is equivalent to

$$
c \cdot\left(y^{i-1}\right)^{n l}=c \cdot\left(x^{i-1}\right)^{l} \in \mathfrak{m}^{n l} S
$$

for some $c \in R$ and for all $l \gg 0$. Since $R$ is normal, Discussion 3.3 shows that $S$ is a free $R$-module. Therefore,

$$
c \cdot\left(x^{i-1}\right)^{l} \in \mathfrak{m}^{n l} .
$$

Thus, $x^{i-1} \in \overline{\mathfrak{m}^{n}}$. Now set $j=i-1$, and choose a minimal reduction $\left(x, x_{2}\right.$, $\left.\ldots, x_{d}\right)$ of $\mathfrak{m}$. Then $x^{j} \in \overline{\left(x, x_{2}, \ldots, x_{d}\right)^{n}}$, and hence $x^{j l} \in\left(x, x_{2}, \ldots, x_{d}\right)^{n(l-t)}$ for some $t$ and for all $l \gg 0$. Since $x, x_{2}, \ldots, x_{d}$ is a system of parameters, this is not possible.

Corollary 3.5. Assume that $(R, \mathfrak{m}, k)$ is a Cohen-Macaulay normal local ring of prime characteristic $p$ which is F-finite with infinite perfect residue field. Let $x \in \mathfrak{m}-\mathfrak{m}^{2}$ be part of a minimal reduction of $\mathfrak{m}$, and let $S=R[y]$ with $y^{n}=x$. Then

$$
e_{H K}(R)-1 \geq \frac{e_{H K}(S)-1}{n} .
$$

Proof. We use Proposition 3.4 and Corollary 2.2. By Proposition 3.4, there is a chain of integrally closed ideals, $\mathfrak{m} S \subset\left(\mathfrak{m} S+\left(y^{n-1}\right)\right) \subset \cdots \subset$ $(\mathfrak{m} S+(y))$, and so using the torsion-free $S$-module $S^{1 / q}$ and applying Corollary 2.2 to the pair of integrally closed ideals $\mathfrak{m} S \subset(\mathfrak{m} S, y)$ yields that $\ell\left(S^{1 / q} / \mathfrak{m} S^{1 / q}\right) \geq(n-1) \operatorname{rank}\left(S^{1 / q}\right)+\ell\left(S^{1 / q} /(\mathfrak{m} S, y) S^{1 / q}\right)$. Dividing by $q^{d}$ and taking limits give that

$$
e_{H K}(\mathfrak{m} S) \geq n+e_{H K}(S)-1 \text {. }
$$

Moreover, $e_{H K}(\mathfrak{m} S)=n \cdot e_{H K}(R)$ by Theorem 3.2. Therefore,

$$
n \cdot e_{H K}(R) \geq n+e_{H K}(S)-1
$$

and hence the result follows.

The last corollary is in some sense our main improvement upon the methods used by Aberbach and Enescu. We use their strategy of adjoining roots of a minimal reduction until we obtain a non- $F$-regular ring; at this point previous estimates are good. The new work of Aberbach and Enescu [AE1], 
which appeared as we were finishing this paper, gives the following result, among other estimates in lower dimensions. Their method of comparing the Hilbert-Kunz multiplicities of radical extensions seems much different from the one we developed above. The new result of Aberbach and Enescu [AE1] is as follows.

THEOREM 3.6 ([AE1]). Let $(R, \mathfrak{m}, k)$ be a local Gorenstein F-regular ring of dimension $d \geq 2$ and Hilbert-Samuel multiplicity $e \geq 6$. Let $k=\mu(\mathfrak{m})-$ $\operatorname{dim}(R)$. Assume further that $R$ is not a complete intersection. Then if $e \geq$ $d !+1$, then $e_{H K}(R) \geq 1+1 / d$ !. Otherwise, if $k=e-2$, then

$$
e_{H K}(R) \geq 1+3\left(\frac{4}{6\left(\left\lceil\frac{d}{2}\right\rceil\right)-2}\right)^{d},
$$

while if $k \neq e-2$, then

$$
e_{H K}(R) \geq 1+\left(\frac{4}{\left(\left\lceil\frac{d}{3}\right\rceil\right) d !+4}\right)^{d}\left(\frac{4}{(6 d-16)}\right) .
$$

Our main result is the following.

Theorem 3.7. Let $(R, \mathfrak{m}, k)$ be a Noetherian local unmixed ring of prime characteristic $p$ which is $F$-finite with infinite perfect residue field and dimension $d \geq 2$. Let $(\boldsymbol{x})$ be a minimal reduction of $\mathfrak{m}$, and let $\mu$ be the maximal number of minimal generators of any ideal in $R /(\boldsymbol{x})$. Let $t$ be the largest integer such that $\overline{\mathfrak{m}^{t}}$ is not contained in $(\boldsymbol{x})$. If $R$ is not regular, then

$$
e_{H K}(R) \geq 1+\left(\min \left\{\frac{1}{d !},\left(\frac{\mu}{e-\mu}\right) \cdot \frac{1}{\left(\left\lceil\frac{d}{t}\right\rceil\right)^{d}}\right\}\right) .
$$

Proof. If $e_{H K}(R) \geq 1+1 / d$ !, there is nothing to prove. Hence, we may assume that $e_{H K}(R)<1+1 / d$ !, and then $R$ is $F$-regular and Gorenstein by [AE, Corollary 3.6]. Thus, we may assume that $R$ is $F$-regular and Gorenstein.

Let $(\boldsymbol{x})=\left(x_{1}, \ldots, x_{d}\right)$ be a minimal reduction of $\mathfrak{m}$, and let $\mu=\mu(\mathfrak{m} /(\boldsymbol{x}))$. Consider the set of overrings $S=R\left[x_{1}^{1 / n}, \ldots, x_{i}^{1 / n}\right]=R_{i, n}$ which are not $F$ regular. Choose $n$ and $i$ such that we attain $\min \left\{n^{i}: R_{i, n}\right.$ is not $F$-regular $\}$. Set $S=R_{i, n}$. Then by Theorem 1.2 applied to $S$, along with the minimal reduction $x_{1}^{1 / n}, \ldots, x_{i}^{1 / n}, x_{i+1}, \ldots, x_{d}$ and the fact that $s(S)=0$ (see Remark 1.4),

$$
e_{H K}(S) \geq \frac{e(S)-s(S) \cdot \mu(S)}{e(S)-\mu(S)}=\frac{e(S)}{e(S)-\mu(S)}
$$


Here, we define $\mu(S)$ to be the maximal number of minimal generators of any ideal in $S /\left(x_{1}^{1 / n}, \ldots, x_{i}^{1 / n}, x_{i+1}, \ldots, x_{d}\right)$.

However, since $S /\left(x_{1}^{1 / n}, \ldots, x_{i}^{1 / n}, x_{i+1}, \ldots, x_{d}\right) \cong R /(\boldsymbol{x})$, we have $e(S)=$ $e(R)$, and $\mu(S)=\mu$. Therefore,

$$
e_{H K}(S) \geq 1+\frac{\mu}{e-\mu}
$$

Let $R_{0}=R$, and for each $i \geq j \geq 1$, let $R_{j}=R_{j-1}\left[x_{j}^{1 / n}\right]$; then by Corollary 3.5 ,

$$
e_{H K}\left(R_{j}\right)-1 \geq \frac{e_{H K}\left(R_{j-1}\right)-1}{n} .
$$

Therefore, we get

$$
e_{H K}(R)-1=e_{H K}\left(R_{0}\right)-1 \geq \frac{e_{H K}(S)-1}{n^{i}} \geq \frac{\mu}{e-\mu}\left(\frac{1}{n^{i}}\right) .
$$

Thus, it remains to prove that

$$
\min \left\{n^{i}: R_{i, n} \text { is not } F \text {-regular }\right\} \leq\left(\left\lceil\frac{d}{t}\right\rceil\right)^{d} .
$$

To do this we note that it suffices to prove that $A=R\left[x_{1}^{1 /\left\lceil\frac{d}{t}\right\rceil}, \ldots, x_{d}^{1 /\left\lceil\frac{d}{t}\right\rceil}\right]$ is not $F$-regular. Set $y_{i}=x_{i}^{1 /\lceil d / t\rceil}, i=1, \ldots, d$. Then a socle representative of $A /(\boldsymbol{x})$ is

$$
u \cdot y_{1}^{\left\lceil\frac{d}{t}\right\rceil-1} \cdots y_{d}^{\left\lceil\frac{d}{t}\right\rceil-1}
$$

where $u$ generates the socle of $(\boldsymbol{x} R)$. By our assumption on $t$, we may in addition assume that $u \in \overline{\mathfrak{m}^{t}}$. Let $v$ be any discrete valuation centered on the maximal ideal of $S$. Then we claim that

$$
v(u)+\left(\left(\left(\left\lceil\frac{d}{t}\right\rceil-1\right) d\right) /\left\lceil\frac{d}{t}\right\rceil\right) v(\mathfrak{m}) \geq d v(\mathfrak{m}) .
$$

Since $v(u) \geq t v(\mathfrak{m})$, it suffices to prove that

$$
t+\left(\left(\left(\left\lceil\frac{d}{t}\right\rceil-1\right) d\right) /\left\lceil\frac{d}{t}\right\rceil\right) \geq d
$$

This simplifies to $t(\lceil d / t\rceil) \geq d$, which is true.

It follows that $u \cdot y_{1}^{\lceil d / t\rceil-1} \cdots y_{d}^{\lceil d / t\rceil-1} \in \overline{(\mathfrak{m} S)^{d}}$. By the tight closure Briançon-Skoda theorem (see $[H H$, Section 5$]$ ), this implies that $\left(x_{1}^{1 /\lceil d / t\rceil}, \ldots\right.$, $\left.x_{d}^{1 /\lceil d / t\rceil}\right) A$ is not tightly closed, which gives the desired conclusion. 
REMARK 3.8. Aberbach and Enescu [AE, p. 15] also state an inequality in their text which is closer to the one we give. Let $r$ be the maximum $i$ such that $\mathfrak{m}^{i}$ is not contained in $(\boldsymbol{x})$. (Note that $r \leq t$.) Let $i_{0}$ be the least integer such that $R_{i}=R\left[x_{1}^{1 / m}, \ldots, x_{i}^{1 / m}\right]$ is not $F$-regular, where $m=\lceil d / r\rceil$. (Observe that $m \geq\lceil d / t\rceil$.) Then

$$
e_{H K}(R) \geq 1+\left(\frac{1}{e(m-1)+1}\right)^{i_{0}}\left(\frac{1}{d}\right) .
$$

In this situation, the estimate is closely related to our estimate above, which we give with a multiple of $1 / n^{i}$; the main difference now is that the estimate of Aberbach and Enescu has an extra $e^{i_{0}}$ in the denominator.

REMARK 3.9. To compare this theorem to the new result of Aberbach and Enescu [AE1], note that since we may assume that $R$ is Gorenstein, $t \geq 2$ unless $R$ is a hypersurface of degree 2 , in which case good bounds are known by [ES]. Moreover, in the notation of Theorem 3.6, if $k \neq e-2$, then $t \geq 3$, and in this case we have essentially removed a factor of $(d !)^{d}$ from the denominator of the estimate given in Theorem 3.6.

REMARK 3.10. We can also improve the statements by bringing in the idea of the core of the maximal ideal, which is the intersection of all reductions of the maximal ideal. The $t$ that we choose in the statement of Theorem 3.7 can actually be chosen maximal so that $\overline{\mathfrak{m}^{t}}$ is not contained in the core of $\mathfrak{m}$; then there will be some minimal reduction which does not contain $\overline{\mathfrak{m}^{t}}$.

Acknowledgment. The authors thank the referee for a careful reading of the paper and for pointing out the statement and proof of Corollary 2.4. The second and third authors gratefully acknowledge support of the National Science Foundation during this research.

\section{REFERENCES}

[AE] I. M. Aberbach and F. Enescu, Lower bounds for Hilbert-Kunz multiplicities in local rings of fixed dimension, Michigan Math. J. 57 (2008), 1-16.

[AE1] - New estimates of Hilbert-Kunz multiplicities for local rings of fixed dimension, preprint, arXiv:1101.5078v2 [math.AC]

[AL] I. M. Aberbach and G. Leuschke, The F-signature and strong F-regularity, Math. Res. Lett. 10 (2003), 51-56.

[BE] M. Blickle and F. Enescu, On rings with small Hilbert-Kunz multiplicity, Proc. Amer. Math. Soc. 132 (2004), 2505-2509.

[BH] W. Bruns and J. Herzog, Cohen-Macaulay Rings, Cambridge Stud. Adv. Math. 39, Cambridge University Press, Cambridge, 1993. 
[Ch] S. Choi, Betti numbers and the integral closure of ideals, Ph.D. dissertation, Purdue University, West Lafayette, Indiana, 1989.

[ES] F. Enescu and K. Shimomoto, On the upper semi-continuity of the Hilbert-Kunz multiplicity, J. Algebra 285 (2005), 222-237.

[GM] I. Gessel and P. Monsky, The limit as $p \rightarrow \infty$ of the Hilbert-Kunz multiplicity of $\sum x_{i}^{d_{i}}$, preprint, arXiv:1007.2004v1 [math.AC]

[HH] M. Hochster and C. Huneke, Tight closure, invariant theory and the BriançonSkoda theorem, J. Amer. Math. Soc. 3 (1990), 31-116.

[HoY] M. Hochster and Y. Yao, Second coefficients of Hilbert-Kunz functions for domains, preprint.

$[\mathrm{H}] \quad$ C. Huneke, Tight Closure and its Applications, with an appendix by Melvin Hochster, CBMS Reg. Conf. Ser. Math. 88, Amer. Math. Soc., Providence, 1996.

[HL] C. Huneke and G. Leuschke, Two theorems about maximal Cohen-Macaulay modules, Math. Ann. 324 (2002), 391-404.

[HMM] C. Huneke, M. A. McDermott, and P. Monsky, Hilbert-Kunz functions for normal rings, Math. Res. Lett. 11 (2004), 539-546.

[HY] C. Huneke and Y. Yao, Unmixed local rings with minimal Hilbert-Kunz multiplicity are regular, Proc. Amer. Math. Soc. 130 (2002), 661-665.

$[\mathrm{Ku}] \quad$ K. Kurano, On Roberts rings, J. Math. Soc. Japan 53 (2001), 333-355.

[M] P. Monsky, The Hilbert-Kunz function, Math. Ann. 263 (1983), 43-49.

[SH] I. Swanson and C. Huneke, Integral Closure of Ideals, Rings, and Modules, London Math. Soc. Lecture Note Ser. 336, Cambridge University Press, Cambridge, 2006.

[T] K. Tucker, F-signature exists, preprint, 2010.

[W] K.-i. Watanabe, "Chains of integrally closed ideals" in Commutative Algebra (Grenoble/Lyon, 2001), Contemp. Math. 331, Amer. Math. Soc., Providence, 2003, 353-358.

[WY1] K.-i. Watanabe and K.-i. Yoshida, Hilbert-Kunz multiplicity and an inequality between multiplicity and colength, J. Algebra 230 (2000), 295-317.

[WY2] - Minimal relative Hilbert-Kunz multiplicity, Illinois J. Math. 48 (2004), 273-294.

[WY3] , Hilbert-Kunz multiplicity of three-dimensional local rings, Nagoya Math. J. 177 (2005), 47-75.

[Y] Y. Yao, Observations on the F-signature of local rings of characteristic p, J. Algebra 299 (2006), 198-218.

Olgur Celikbas

Department of Mathematics

University of Kansas

Lawrence

Kansas 66045-7523

USA

ocelikbas@math.ku.edu 
Hailong Dao

Department of Mathematics University of Kansas

Lawrence

Kansas 66045-7523

$U S A$

hdao@math.ku.edu

Craig Huneke

Department of Mathematics University of Kansas

Lawrence

Kansas 66045-7523

USA

huneke@math.ku.edu

Yi Zhang

Department of Mathematics University of Minnesota

Minneapolis

Minnesota 55455

USA

zhang397@umn.edu 